\title{
Optimizing the dose of intrathecal morphine when combined with continuous $3-$ in -1 nerve block after total knee replacement
}

\author{
Department of Anesthesiology and Pain Medicine, College of Medicine, Eulji University, *Konyang University, Daejeon, Korea
}

\author{
Chang Kil Park, Choon Kyu Cho*, Jong Hyuk Lee, and Hyun Ho Shin
}

\begin{abstract}
Background: Continuous femoral 3-in-1 block alone is insufficient for the treatment of severe pain after total knee replacement (TKR). Intrathecal (IT) morphine provides effective postoperative analgesia but may result in many side effects. The optimal dose of spinal morphine when combined with continuous 3-in-1 block after TKR is not known.

Methods: Patients were randomized to receive IT morphine in five groups ( $\mathrm{n}=20$ per group): 1) $0.0 \mathrm{mg}, 2) 0.05 \mathrm{mg}, 3) 0.1$ $\mathrm{mg}$, 4) $0.15 \mathrm{mg}$, and 5) $0.2 \mathrm{mg}$. All patients received continuous 3-in-1 block performed with $20 \mathrm{ml}$ of $0.25 \%$ bupivacaine, followed by a continuous infusion of $0.125 \%$ bupivacaine at the rate of $2 \mathrm{ml} / \mathrm{h}$ plus PCA boluses of $1 \mathrm{ml}$ with a lockout of 10 minutes. The intensity of pain at rest and on movement of the knee was assessed by using a visual analog scale for the first two postoperative days.

Results: All treatment groups produced effective pain relief and decreased cumulative femoral PCA bolus use of $0.125 \%$ bupivacaine compared with control, respectively $(\mathrm{P}<0.05)$; however, there were no significant differences among the treatment groups. The incidence of vomiting was significantly more frequent with $0.1-0.2 \mathrm{mg}$ IT morphine groups compared with control, respectively (P $<0.05)$. The rate of administration of antipruritic medication was increased as IT morphine dose increased $(\mathrm{P}<0.05)$.

Conclusions: Use of $0.05 \mathrm{mg}$ IT morphine would appear to provide the optimal balance between pain relief and adverse effects following TKR. (Korean J Anesthesiol 2009; 57: 69 77)
\end{abstract}

Key Words: Intrathecal morphine, Visual analog scale, 3-in-1 block.

\section{INTRODUCTION}

Total knee replacement (TKR) is associated with marked and prolonged postoperative pain. In fact, TKR is a more painful procedure than total hip replacement and posterolateral lumbar spinal fusion [1-3]. Achieving adequate analgesia remains a clinical challenge. After TKR, postoperative pain relief can be achieved by several approaches, such as IV patient-controlled analgesia (PCA), epidural analgesia, and femoral 3-in-1 nerve

Received: December 30, 2008.

Accepted: May 20, 2009.

Corresponding author: Chang Kil Park, M.D., Department of Anesthesiology and Pain Medicine, Eulji University Hospital, 1306, Dunsan-dong, Seo-gu, Daejeon 302-120, Korea. Tel: 82-42-611-3651, Fax: 82-42-482-9036, E-mail: pck@eulji.ac.kr

Copyright (C) Korean Society of Anesthesiologists, 2009

(C) This is an open-access article distributed under the terms of the Creative Commons Attribution License, which permits unrestricted use, distribution, and reproduction in any medium, provided the original work is properly cited. block [4,5]. Epidural infusions containing local anesthetics (with or without an opioid) provide superior analgesia, but are associated with arterial hypotension, urinary retention, and difficulty with ambulation because of bilateral motor block [5]. In addition, epidural catheterization in the presence of thromboprophylaxis increases the risk of neuraxial hematoma [6]. Continuous 3-in-1 block is as efficient as epidural analgesia and induces fewer side effects, and is therefore, the preferred technique [5]. However, the pain in the back of the knee after TKR was not relieved by 3-in-1 block [7]. This suggests that both the obturator and the sciatic nerve also provide a major contribution to the innervation of the knee joint $[8,9]$.

Although speculative, intrathecal (IT) morphine use will simultaneously provide analgesia for the sciatic nerve distribution and maintain an acceptable level of side effects. A single small dose of IT morphine would be coupled with the femoral nerve block as part of a multimodal analgesia in TKR. Recently, the authors [10] determined that the addition of a smaller dose, $0.1 \mathrm{mg}$, of IT morphine to continuous 3-in-1 
block improves postoperative analgesia compared with 3-in-1 block alone after TKR. We assumed that if combined with 3-in-1 block, the effective dose of IT morphine could be reduced to a smaller one than those speculated by Tarkkila et al $(0.3 \mathrm{mg})$ [11] and Rathmell et al $(0.2 \mathrm{mg})$ [2]. However, the optimal dose and adverse effects of spinal morphine when combined with continuous 3-in-1 block after TKR is not known. Furthermore, previous dose-ranging studies with IT morphine have been performed in hip arthroplasty [12,13] or combining TKR and open ligament surgery [11] or combining hip and knee arthroplasty [14], but not performed in only TKR. Rathmell et al [2] suggested that pain after knee arthroplasty was more intense than that after hip arthroplasty. Thus, the practice of combining TKR and other types of major joint surgery in the evaluation of an outcome is not valid as in the study of Tarkkila et al [11] and Jacobson et al [14].

IT morphine is an effective, convenient, and simple method for management of postoperative pain but may result in many side effects, including postoperative nausea and vomiting (PONV), pruritus, and notably delayed respiratory depression [14-17]. Furthermore, older patients may be particularly sensitive to the respiratory-depressant effects of IT morphine [18]. The patients in previous studies had a mean age of 65 [2], 66 [11], and 67 year [10] in major knee surgery. Therefore, a minimum effective dose of IT morphine for TKR while avoiding respiratory depression is needed.

We hypothesized that the analgesic dose and side effects of IT morphine when combined with 3 -in-1 block might be further reduced than those seen in previous studies [2,11]. Accordingly, we performed a trial to establish a minimum effective dose of IT morphine after doses of 0.0, 0.05, 0.1, 0.15, and $0.2 \mathrm{mg}$ and to evaluate side effects when combined with continuous 3-in-1 block after unilateral TKR.

\section{MATERIALS AND METHODS}

After informed consent and with institutional approval, 123 ASA physical status I or II patients scheduled for elective unilateral TKR under spinal anesthesia (SA) were enrolled, and those patients who received the surgery in the morning were included in this study. Patients were excluded if they met any of the following criteria: contraindications to regional anesthetic technique (e.g., local infection, sepsis, coagulation abnormality), allergy to local anesthetic and/or opioid, pre-existing neurological deficit in the lower extremity, or inability to comprehend pain scales or to use a PCA device. After surgery in the postanesthesia care unit (PACU), all patients were given an explanation of visual analog scale (VAS) and were familiarized with the use of PCA connected to the femoral catheter. No significant differences were found among the groups in terms of age, gender distribution, weight, height, anesthetic time, and operating time (Table 1).

We designed this prospective study to evaluate the analgesic efficacy and side effect profile of $0.0-0.2 \mathrm{mg}$ of IT morphine when combined with continuous 3-in-1 block after TKR. Patients were randomized to receive IT morphine in five groups (n = 20 per group): 1) $0.0 \mathrm{mg}, 2) 0.05 \mathrm{mg}$, 3) $0.1 \mathrm{mg}$, 4) $0.15 \mathrm{mg}$, and 5) $0.2 \mathrm{mg}$. Premedication consisted of $1-2 \mathrm{mg}$ of IV midazolam. SA was performed, with the patient in the lateral position, at the L3-4 or 4-5 intervertebral space by using a 25-gauge Quinke needle. All patients were administered 6-13 mg of hyperbaric bupivacaine $0.5 \%$ and epinephrine $0.05-0.1 \mathrm{mg}$ together with $0.0-0.2 \mathrm{mg}$ of morphine into the IT space, depending on group allocation. The exact doses of bupivacaine and epinephrine used were left to the discretion of the attending anesthesiologist. Further intraoperative sedation consisted of midazolam in 1-mg increments. All patients re-

Table 1. Demographic and Perioperative Data

\begin{tabular}{lccccc}
\hline & & \multicolumn{4}{c}{ IT morphine } \\
\cline { 3 - 6 } & & $0.05 \mathrm{mg}$ & $0.1 \mathrm{mg}$ & $0.15 \mathrm{mg}$ & $0.2 \mathrm{mg}$ \\
\hline Age (yr) & $68.6 \pm 6.3$ & $69.9 \pm 7.9$ & $69.7 \pm 7.1$ & $68.7 \pm 5.3$ & $67.4 \pm 7.0$ \\
Sex (M/F) & $1 / 19$ & $1 / 19$ & $1 / 19$ & $2 / 18$ & $0 / 20$ \\
Weight (kg) & $62.6 \pm 10.9$ & $59.7 \pm 9.5$ & $61.6 \pm 10.6$ & $62.3 \pm 8.7$ & $63.5 \pm 10.9$ \\
Height (cm) & $151.6 \pm 5.9$ & $148.8 \pm 5.5$ & $152.1 \pm 8.2$ & $151.0 \pm 7.2$ & $149.8 \pm 6.4$ \\
Operation time (min) & $104.8 \pm 22.1$ & $99.8 \pm 20.3$ & $106.0 \pm 17.7$ & $100.3 \pm 19.4$ & $92.5 \pm 18.6$ \\
Anesthesia time (min) & $179.3 \pm 30.5$ & $174.8 \pm 35.8$ & $180.8 \pm 33.8$ & $172.0 \pm 31.7$ & $156.3 \pm 30.8$ \\
\hline
\end{tabular}

All values except gender ratio are expressed as mean \pm SD. IT: intrathecal. 
ceived oxygen $3 \mathrm{~L} / \mathrm{min}$ via nasal cannula for the duration of the procedure. Standard monitoring, including noninvasive blood pressure, electrocardiogram, and oxygen saturations, was used in all patients for the duration of surgery. Surgery was performed with a tourniquet applied in the thigh and inflated at $300 \mathrm{mmHg}$.

The femoral catheters for continuous 3-in-1 block were placed before the induction of SA by one of the authors experienced in the techniques. With patients in a supine position, the pulse of the femoral artery was identified. The needle entry site was at the level of the inguinal skin crease $1-2 \mathrm{~cm}$ lateral to the femoral pulse. The femoral nerve was accurately located with a peripheral nerve stimulator $\left(\right.$ Innervator ${ }^{\circledR}$, Fisher \& Paykel, New Zealand) connected to an 18-gauge short-beveled cannula (Contiplex ${ }^{\circledR}$ set; B. Braun, Melsungen, Germany). With a starting output of $2.0 \mathrm{~mA}$ at $1 \mathrm{~Hz}$ frequency, the needle was advanced cephalad in a sagittal plane at an angle of $30-40^{\circ}$ to the skin until twitches of the quadriceps femoris muscle (ascension of the patella) were elicited. Its position was then judged adequate when output lower than $0.4 \mathrm{~mA}$ still elicited contractions of the quadriceps. A 20-gauge catheter was threaded $10-15 \mathrm{~cm}$ into the psoas compartment. Catheters were secured to the skin with catheter clamps (Lockit, Portex, Hythe, UK), and then it was covered with a transparent dressing (Ioban $^{\mathrm{TM}} 2$, 3M Health Care, USA).

To verify the correct position of the femoral catheter, the 3 $\mathrm{ml}$ of contrast media (iopamidol) was injected via the catheter and the location of the catheter was confirmed with $\mathrm{C}$-arm machine. At the end of operation, a $20 \mathrm{ml}$ bolus of $0.25 \%$ bupivacaine with epinephrine $1: 200,000$ was administered into the catheter after a negative aspiration test for blood. During the injection, firm pressure was manually applied just distally to the catheter entry point to encourage the cephalad spread of the local anesthetic. One hour after the bolus injection, a continuous infusion of $0.125 \%$ bupivacaine at the rate of $2 \mathrm{ml} / \mathrm{h}$ plus PCA boluses of $1 \mathrm{ml}$ with a lockout of 10 minutes was started, which then continued into the postoperative period for 3 or 4 days. The amount of postoperative bupivacaine received by each patient for the first two days was noted and the femoral catheters were removed on the 3 rd or 4th postoperative day (POD).

The intensity of pain at rest and with a standardized motion of the knee $\left(30^{\circ}\right.$ of passive flexion) was assessed by the patients using a VAS $(0=$ no pain, $100=$ worst possible pain $)$, one hour after arrival to the PACU, at $6 \mathrm{PM}$ on the day of operation, and at $9 \mathrm{AM}$ and $6 \mathrm{PM}$ on POD 1 and 2, respectively. Sensory block by using a pinprick test with a blunt needle in the distributions of the femoral (anterior aspect of the thigh), the obturator (inner aspect of the thigh), and the lateral femoral cutaneous nerve (outer aspect of the thigh) were assessed in the middle third of the thigh twice a day by an independent observer. It was confirmed by reduced sensation involving the distribution of each nerve compared with contralateral thigh.

For postoperative analgesia, diclofenac (90 mg two times daily) was regularly administered IM to all patients on arrival to the ward and for the first two days. If the VAS was $>50$ or the patient requested, $90 \mathrm{mg}$ of diclofenac or $0.5 \mathrm{mg}$ of butorphanol IM was supplemented in both groups. The type of analgesic used was left to the treating physician. Ten mg metoclopramide IV and $8 \mathrm{mg}$ ondansetron IV were routinely prescribed by orthopedic surgeon for antiemetic medications to all patients on arrival to the ward and for the first two days. Treatment for pruritus consisted of IM chlorpheniramine $4 \mathrm{mg}$, and treatment in all cases was initiated at the patient's request. Naloxone was reserved for treatment of pruritus that was resistant to chlorpheniramine therapy. Supplemental analgesia, antiemetic and antipruritic medications, and side effects were recorded for each group at each measuring interval.

The presence and severity of nausea was assessed by using an ordinal scale $(0=$ no nausea; $1=$ mild; $2=$ moderate; $3=$ severe).

All patients in this series were monitored for respiratory depression by assessment of their respiratory rate, which is the means routinely used to monitor patients after IT morphine in our institution. Respiratory rates were assessed by the study anesthesiologist at the measurement times and also by the ward nurses every four hours. Respiratory frequency was measured with the patients at rest and undisturbed, and prior to making the remaining observations. The incidence of respiratory depression, defined as respiratory rate $<10$ breaths/min, was recorded. Supplemental oxygen was not placed routinely; however, if at any time their respiratory rate was $<10$ breaths/min, oxygen $3 \mathrm{~L} / \mathrm{min}$ via nasal cannula was added. Intravenous naloxone in $0.1 \mathrm{mg}$ increments was to be given for treatment of respiratory depression.

Sedation was scored according to the following scale: $0=$ alert; 1 = drowsy; 2 = sleeping, easy to arouse; and $3=$ sleeping, difficult to arouse. Significant sedation was defined as a sedation score of 3 . Urinary retention could not be assessed 
because indwelling catheters in the urinary bladder were left in place for approximately 24 hours postoperatively. All data were collected by an anesthesiologist not involved in the administration of anesthesia nor in patient care in the recovery room.

Statistical analysis was performed with the SPSS software (version 12.0, USA). To compare patient's age, weight and height, anesthetic time, and operating time, the one-way ANOVA test was performed and the Turkey HSD test was used as post hoc analysis. The one-way ANOVA and Turkey HSD test as post hoc test were used to compare PCA bolus use and VAS pain scores. A chi-squared or Fisher exact test was used to compare gender, side effects and rescue antipruritics. A chi-square for trend test was used to determine whether the incidence of PONV, pruritus, antipruritic therapy, and sedation score had statistically significant ordering based on the dose of IT morphine. A P value $<0.05$ was considered significant.

\section{RESULTS}

A total of 123 patients were entered into the study. Of these patients, 23 were excluded from the data analysis: femoral nerve block failed despite successful identification of the femoral nerve with a nerve stimulator in six; in 13 patients who had successful initial 3-in-1 block after injection of $0.25 \%$

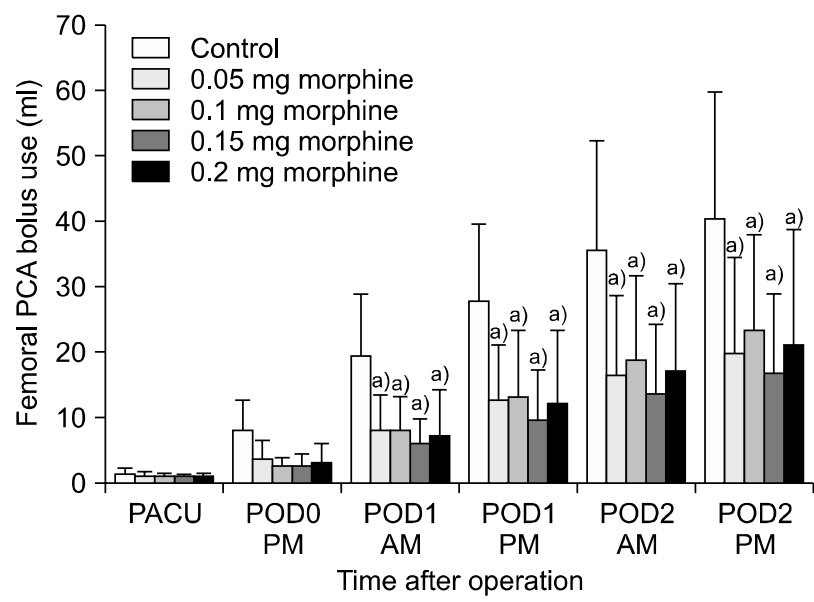

Fig. 1. Cumulative femoral PCA bolus use of $0.125 \%$ bupivacaine. All IT morphine groups produced decreased cumulative PCA bolus use of $0.125 \%$ bupivacaine compared with control on POD 1 and 2 , respectively; however, there were no significant differences among treatment groups. PCA: patient-controlled analgesia, PACU: postanesthesia care unit, POD: postoperative day. ${ }^{\text {a) }} \mathrm{P}<0.05$ when compared with control. bupivacaine $20 \mathrm{ml}$, the sensory block disappeared in the ward despite continuous infusion of $0.125 \%$ bupivacaine; femoral catheter was accidentally dislodged by patients on POD 1 in three; recording of bolus use of $0.125 \%$ bupivacaine was incomplete on POD 2 in one. If a patient was removed from the trial, the same trial was subsequently performed in another patient. The final total of 100 patients were equally distributed among the groups.

The four treatment groups that received IT morphine produced decreased cumulative PCA bolus use of $0.125 \%$ bupivacaine for the first two days compared with control $(\mathrm{P}<0.05)$ (Fig. 1). However, there were no significant differences among any of the treatment groups.

The VAS pain scores at rest were significantly lower in treatment groups than control at $6 \mathrm{PM}$ on the day of operation and at $9 \mathrm{AM}$ on POD 1, respectively $(\mathrm{P}<0.05)$; however, there were no significant differences among treatment groups (Fig. 2). Pain scores on movement were significantly lower in $0.05,0.15$, and $0.2 \mathrm{mg}$ IT morphine groups than control at 6 $\mathrm{PM}$ on the day of operation, respectively ( $\mathrm{P}<0.05$ ); however, there were no significant differences among treatment groups (Fig. 3). Pain scores on movement in $0.1 \mathrm{mg}$ IT morphine were also lower than control at $6 \mathrm{PM}$ on the day of operation, but this difference did not reach statistical significance. By contrast, at 6 PM on POD 2, pain scores on movement were

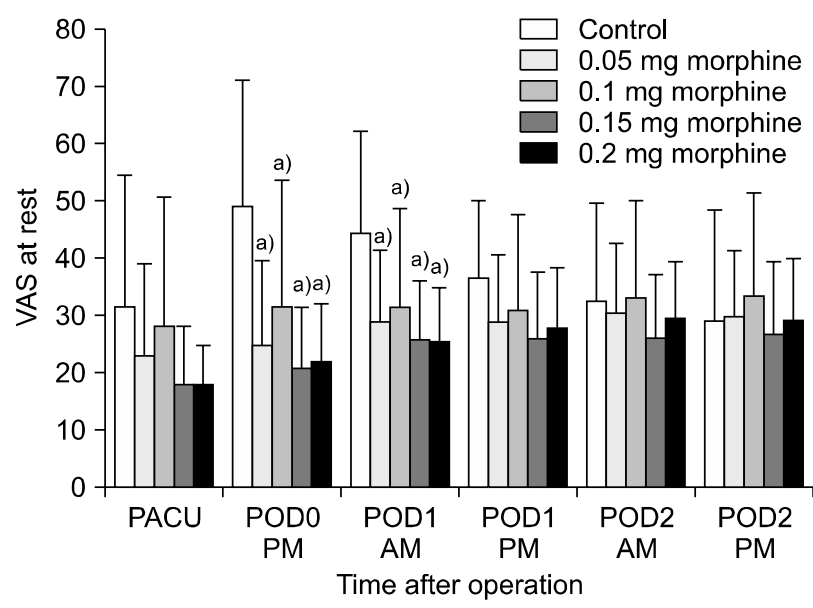

Fig. 2. VAS pain scores at rest. The VAS scores at rest were significantly lower in all IT morphine groups than control at $6 \mathrm{PM}$ on the day of operation and at 9 AM on POD 1, respectively; however, there were no significant differences among treatment groups. VAS: visual analog scale, PACU: postanesthesia care unit, POD: postoperative day. ${ }^{\text {a) }} \mathrm{P}<0.05$ when compared with control. 
significantly higher in 0.05 and $0.1 \mathrm{mg}$ IT morphine groups than control, respectively ( $\mathrm{P}<0.05$ ); however, there were no significant differences among treatment groups (Fig. 3).

The incidence of nausea was significantly more frequent in $0.15 \mathrm{mg}$ IT morphine than control $(\mathrm{P}<0.05)$ (Table 2$)$. The incidence of vomiting was significantly more frequent in 0.1 ,

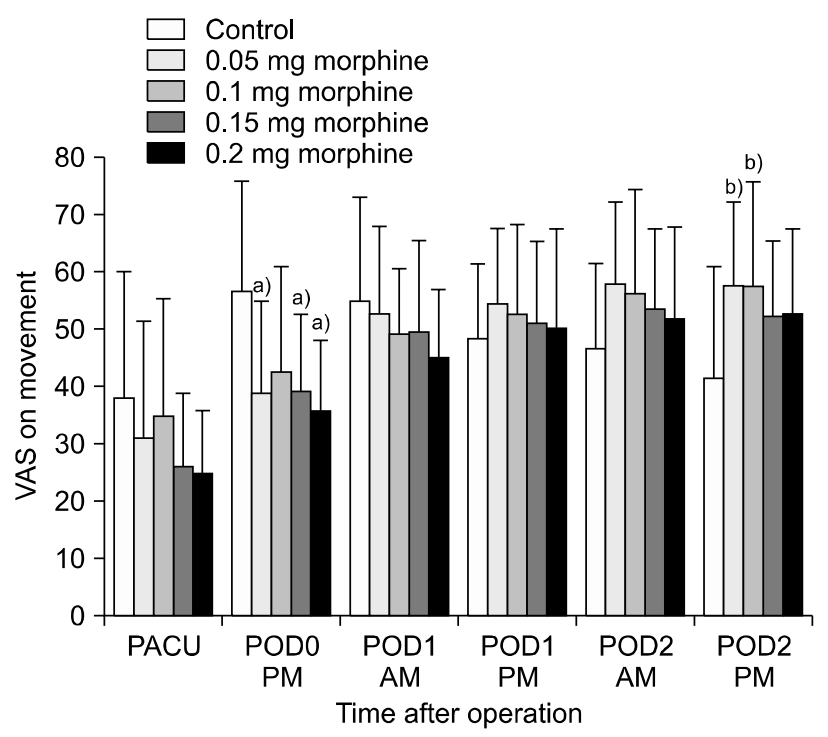

Fig. 3. VAS pain scores on movement. The VAS scores on movement were significantly lower in $0.05,0.15$, and $0.2 \mathrm{mg}$ IT morphine than control at $6 \mathrm{PM}$ on the day of operation, respectively; however, there were no significant differences among treatment groups. The VAS scores on movement were significantly higher in 0.05 and $0.1 \mathrm{mg}$ IT morphine than control at $6 \mathrm{PM}$ on POD 2, respectively; however, there were no significant differences among treatment groups. VAS: visual analog scale, PACU: postanesthesia care unit, POD: postoperative day. ${ }^{\text {a) }} \mathrm{P}<0.05$ when compared with control. ${ }^{b} \mathrm{P}<0.05$ when compared with control.
0.15 , and $0.2 \mathrm{mg}$ IT morphine groups than control $(\mathrm{P}<0.05)$; however, there were no significant differences among treatment groups (Table 2).

The incidence of pruritus was significantly more frequent in treatment groups than control $(\mathrm{P}<0.05)$; however, there were no significant differences among treatment groups (Table 2). For the rate of administration of antipruritic medication, there were significant differences between control and treatment groups $(\mathrm{P}<0.05)$. Although no significant difference among treatment groups was observed regarding the rate of antipruritic therapy, a significant trend towards an increasing number of patients who required antipruritic therapy was found as IT morphine dose increased $(\mathrm{P}<0.05)$ (Table 2). Most patients suffering from pruritus were treated with chlorpheniramine successfully. However, four patients who were resistant to chlorpheniramine therapy received naloxone $0.2-0.4 \mathrm{mg} ; 2$ each in 0.05 and $0.15 \mathrm{mg}$ IT morphine groups.

The incidence of sedation score $=1$ was significantly more frequent in 0.15 and 0.2 IT morphine groups than control $(\mathrm{P}<$ 0.05 ) (Table 2). No patient showed sedation score $=3$, accordingly a significant sedation was not found in all patients. There was no patient with respiratory rate $<10$ breaths/min or sleep apnea. No patient needed additional oxygen in the ward. Consequently, no patient showed any evidence of respiratory depression.

Not all 3-in-1 blocks were successful; complete 3-in-1 block was $83.2 \%$ of all patients. There was no complication of prolonged anesthesia, infection, or damage to the femoral nerve from the continuous 3-in-1 block procedure.

All IT morphine groups provided comparable and effective postoperative analgesia. However, the incidence of vomiting

Table 2. Incidence of Nausea, Vomiting, Pruritus and Sedation

\begin{tabular}{|c|c|c|c|c|c|c|}
\hline & & \multirow{2}{*}{ Control } & \multicolumn{4}{|c|}{ IT morphine } \\
\hline & & & $0.05 \mathrm{mg}$ & $0.1 \mathrm{mg}$ & $0.15 \mathrm{mg}$ & $0.2 \mathrm{mg}$ \\
\hline \multirow[t]{4}{*}{ Nausea score } & 0 & $13(65)$ & $6(30)$ & $8(40)$ & $4(20)^{a)}$ & $9(45)$ \\
\hline & 1 & $7(35)$ & $10(50)$ & $6(30)$ & $10(50)$ & $6(30)$ \\
\hline & 2 & $0(0)$ & $4(20)$ & $5(25)$ & $4(20)$ & $4(20)$ \\
\hline & 3 & $0(0)$ & $0(0)$ & $1(15)$ & $2(10)$ & $1(5)$ \\
\hline Vomiting & & $2(10)$ & $7(35)$ & $11(55)^{\mathrm{a})}$ & $9(45)^{a)}$ & $9(45)^{a)}$ \\
\hline Pruritus & & $0(0)$ & $13(65)^{\mathrm{a})}$ & $14(70)^{\mathrm{a})}$ & $15(75)^{a)}$ & $16(80)^{a)}$ \\
\hline Rescue antipruritic & & $0(0)$ & $7(35)^{\mathrm{a}, \mathrm{b})}$ & $8(40)^{\mathrm{a}, \mathrm{b})}$ & $9(45)^{\mathrm{a}, \mathrm{b})}$ & $14(70)^{\mathrm{a}, \mathrm{b})}$ \\
\hline \multirow{2}{*}{ Sedation score } & 0 & $20(100)$ & $16(80)$ & $16(80)$ & $12(60)$ & $13(65)$ \\
\hline & 1 & $0(0)$ & $4(20)$ & $4(20)$ & $8(40)^{a)}$ & $7(35)^{\mathrm{a})}$ \\
\hline
\end{tabular}

Date are number $(\%)$ of patients. IT: intrathecal. ${ }^{a} \mathrm{P}<0.05$ compared with control group. A significant trend towards increasing number of patients who required antipruritic therapy was found as IT morphine dose increased $\left({ }^{\mathrm{b})} \mathrm{P}<0.05\right)$. 
was significantly more frequent with $0.1-0.2 \mathrm{mg}$ IT morphine groups compared with control. The rate of administration of antipruritic medication was increased as IT morphine dose increased. Therefore, $0.05 \mathrm{mg}$ IT morphine would appear to provide the optimal balance between pain relief and adverse effects following TKR.

\section{DISCUSSION}

Our study demonstrates that all IT morphine groups provided comparable and effective postoperative analgesia when combined with continuous 3-in-1 block after TKR. Indeed, administration of $0.05-0.2 \mathrm{mg}$ IT morphine showed no clear dose-response relationship relating to analgesic efficacy. Our experience indicates that the minimum effective dose of IT morphine appears to be as low as $0.05 \mathrm{mg}$. However, the incidence of vomiting was significantly more frequent with $0.1-$ $0.2 \mathrm{mg}$ IT morphine groups compared with control. The rate of administration of antipruritic medication was increased as IT morphine dose increased. Therefore, $0.05 \mathrm{mg}$ IT morphine would appear to provide the optimal balance between pain relief and adverse effects following TKR.

A multimodal approach to pain control with the use of a combination of drugs or a multiple analgesic method that relieve pain by different mechanisms has been widely proposed in the literature [19]. Whereas the main site of action of subarachnoid morphine is the opioid receptors in the substantia gelatinosa of the dorsal horn of the spinal cord [20], femoral nerve block has peripheral effects, as it blocks the massive afferent nociceptive input [5]. After knee surgery, postoperative pain can be associated with reflex spasm of the quadriceps muscle, causing further pain and impaired muscle function. Abolition of quadriceps muscle spasm by femoral nerve block contributes to the effectiveness of the technique [4].

IT morphine is effective for acute postoperative pain control in patients undergoing a number of different types of surgery. In an earlier study, Jacobson et al [14] demonstrated that low dose $(0.3 \mathrm{mg})$ was not effective, but larger doses (1.0 and 2.5 $\mathrm{mg}$ ) provided reliable and prolonged analgesia in patients undergoing total knee or hip replacement. However, respiratory depression or apnea was common after the administration of larger doses. Thus, doses of IT morphine should be as small as possible to reduce the side-effects. Kalso [21] found that small doses of IT morphine $(0.2 \mathrm{mg})$ were not as effective as larger doses $(0.4 \mathrm{mg})$ in providing prolonged postoperative an- algesia after orthopedic surgery. Likewise, Tarkkila et al [11] found that $0.3 \mathrm{mg}$ IT morphine alone was insufficient for the treatment of severe pain after major knee surgery (TKR or open ligament surgery). Magnitude and duration of analgesia after IT morphine were dose-related and increases in $\mathrm{PaCO}_{2}$ were also significantly related to IT morphine dose [16]. Indeed, it appears that the efficacy of doses above $0.3 \mathrm{mg}$ IT morphine is often limited by side effects [22]. Accordingly, there is no ideal dose of IT morphine that provides superior analgesia free from adverse ventilatory effects. One generalization emerges that larger doses of IT morphine provide excellent analgesia, but were often accompanied by respiratory depression or apnea, whereas smaller doses producing little respiratory effect provide inadequate analgesia. Therefore, smaller doses of IT morphine should be used as one part of multimodal analgesia for the treatment of severe pain. In our study, smaller doses of IT morphine were combined with 3-in-1 block as a multimodal analgesia protocol for TKR.

Various dose-ranging studies have been performed to seek smaller effective doses of IT morphine. Rathmell et al [2] demonstrated that combining 0.2 or $0.3 \mathrm{mg}$ IT morphine with PCA morphine provides good to excellent pain control than those receiving 0.0 or $0.1 \mathrm{mg}$ after total hip or knee arthroplasty. Slappendel et al [12] studied the analgesic effect of $0.025,0.05,0.1,0.2 \mathrm{mg}$ IT morphine for total hip surgery and believed that $0.1 \mathrm{mg}$ of IT morphine provides excellent postoperative analgesia in the first 24 hours, and IT morphine doses $<0.1 \mathrm{mg}$ were less effective. Murphy et al [13] also reported that both 0.1 and $0.2 \mathrm{mg}$ of IT morphine with PCA morphine provide effective postoperative analgesia after hip arthroplasty, whereas $0.05 \mathrm{mg}$ of IT morphine did not provide effective analgesia compared with placebo. Likewise, Uchiyama et al [23] reported that the analgesic effect of $0.1 \mathrm{mg}$ was seen to be greater than that of $0.05 \mathrm{mg}$ and similar to that of $0.2 \mathrm{mg}$ of IT morphine, indicating that $0.1 \mathrm{mg}$ is very likely the minimum effective dose for caesarean section. Accordingly, the optimal doses of IT morphine in the major arthroplasty surgery and caesarean section have been shown to be 0.1 or $0.2 \mathrm{mg}[2,12,13,23]$. However, in our study the minimum effective dose of IT morphine was as low as $0.05 \mathrm{mg}$. A possible explanation for the different results may be as follows: first, in our study, a significant analgesia might have been achieved in part with the 3-in-1 block and therefore any meaningful comparisons could not be obtained in the VAS pain scores among treatment groups; second, the dose range of IT 
morphine from 0.05 to $0.2 \mathrm{mg}$ used was probably too small to allow any meaningful comparisons for the VAS pain scores.

Pain assessment should be made for patients both at rest and during activity. Because activity is often an important part of the rehabilitation of the surgical patient, it is critical that one assess movement pain [24]. Pain scores with mobilization may provide additional useful information. First, in our study, no difference was noted among treatment groups in VAS scores at rest at $6 \mathrm{PM}$ on POD 2, whereas VAS scores on movement were significantly higher in 0.05 and $0.1 \mathrm{mg}$ IT morphine than control at the same measurement time, respectively. However, there was no statistical significance in VAS scores on movement in 0.15 and $0.2 \mathrm{mg}$ IT morphine compared with control at the same measurement time, respectively. This might be explained by the fact that both 0.15 and $0.2 \mathrm{mg}$ had longer duration of action compared with 0.05 and $0.1 \mathrm{mg}$ IT morphine. Similar dose-response observation has been reported by Chadwick and Ready [25] in which there appeared to be a tendency for a long duration of analgesia with increasing doses of IT morphine $(0.3-0.5 \mathrm{mg})$. Second, in our study, there may be a trend toward decreasing VAS scores at rest and on movement over time in control, whereas a trend toward increasing VAS scores on movement over time in treatment groups. In the postoperative period, hyperalgesia can occur either due to nervous system sensitization by surgical stimuli (nociception-induced hyperalgesia) or to an effect of drugs used (drug-induced hyperalgesia), particularly analgesics such as $\mu$-opioid agonists [26]. Even a single opioid administration induces a short-lasting analgesic effect followed by a delayed anti-analgesic or hyperalgesic effect [27]. Therefore, it seems that drug-induced hyperalgesia by IT morphine used may lead to a trend toward increasing VAS scores on movement over time in treatment groups in our study.

The emesis is one of the more distressing adverse effects of IT morphine. In our study, there was a significant difference in the incidence of nausea only between $0.15 \mathrm{mg}$ IT morphine and control. The incidence of vomiting was significantly more frequent with $0.1,0.15$, and $0.2 \mathrm{mg}$ IT morphine than control, however, there was no evidence of a difference among treatment groups. This could be explained by the fact that this dosage range might be too small to allow any meaningful comparisons, and routine postoperative antiemetic medications might influence the results. Slappendel et al [12] demonstrated that PONV showed similar overall incidences in all IT morphine groups $(0.025-0.2 \mathrm{mg})$ and that PONV was not in- duced by these small doses of IT morphine. Similar findings were observed by Murphy et al [13], who showed no significant difference in the incidence or severity of PONV in the groups that received IT morphine $(0.05-0.2 \mathrm{mg})$ compared with the control.

Pruritus may contribute to patient discomfort. In our study, no difference in the incidence of pruritus among treatment groups was noted. However, a significant trend towards an increasing number of patients who required antipruritic therapy was found as IT morphine dose increased. The need for antipruritic therapy is an index of the severity of the pruritus [13]. Accordingly, we found that the incidence of itching was not dose related, but dose related in the severity of pruritus. To examine our data, there may be a trend toward increasing the duration of pruritus as IT morphine dose increased (data not shown). Similar findings was noted by Kuipers et al [28] who showed the longer duration of pruritus with $0.3 \mathrm{mg}$ (maximum $22 \mathrm{~h}$ ) versus $0.1 \mathrm{mg}$ IT morphine (maximum $15 \mathrm{~h}$ ). Meanwhile, Slappendel et al [12] found that the incidence of itching was dose related, as was the incidence of request for antipruritic medication in the dose range of $0.025-0.2 \mathrm{mg}$ of IT morphine. Murphy et al [13] also reported that the overall incidence of pruritus and request for antipruritic therapy was significantly increased in patients who received $0.2 \mathrm{mg}$ IT morphine compared with 0.05 and $0.1 \mathrm{mg}$ IT morphine groups.

The incidence of sedation score of 1 was significantly more frequent with treatment groups than control in our study. To examine our data, none of patients showed a sedation score of 2 or 3 , therefore there was no evidence of deep sedation at any of the doses of IT morphine studied. Likewise, Murphy et al [13] found that sedation did not appear to be a problem at the doses of IT morphine $(0.05-0.2 \mathrm{mg})$ used in their study.

Delayed respiratory depression is the most feared side effect of IT morphine and has restricted its use as an usual component of postoperative analgesia. Although the respiratory rate did not reliably indicate hypoxemia [16], there was no patient with a respiratory rate $<10$ breaths/min in our study. The most reliable clinical sign of significant respiratory depression appears to be a depressed level of consciousness [29]. No patient showed a sedation score of 2 or 3 ; thus, a significant sedation was not found in all patients given IT morphine in our study. Therefore, it can be assumed that there was no patient with significant respiratory depression and who required oxygen therapy in our study.

Clinicians continue to hesitate to prescribe IT morphine be- 
cause of the serious nature of opioid-induced respiratory depression. The safest course, therefore, would seem to be to give the minmum effective dose hoping to minimize the possibility of respiratory depression. Although close surveillance of breathing is mandatory there is increasing recognition that low dose neuraxial administration does not obligate patients to be observed in an intense monitoring environment. Glass [15] recommended that doses of possibly $0.05-0.1 \mathrm{mg}$ IT morphine may well be as effective and much safer. Slappendel et al [12] also believed that $0.1 \mathrm{mg}$ of IT morphine does not cause significant respiratory depression and monitoring in intensive care units is not required because of the administration of this IT morphine dose. Likewise, Schaer et al [30] found that no particular supervision due to the administration of IT morphine is necessary in the dose range from $0.06-0.08 \mathrm{mg}$ if systemic opiates are avoided. One generalization emerges that respiratory depression is reported to occur only if doses $\geq 0.15 \mathrm{mg}$ are used, whereas it is very rare for doses of $0.1 \mathrm{mg}$ IT morphine or less if not combined with intravenous morphine. In our findings, none of the patients who received $0.05-0.2 \mathrm{mg}$ of IT morphine developed respiratory depression. Furthermore, the minimum effective dose of IT morphine was $0.05 \mathrm{mg}$, and hence it can be assumed that the risk of respiratory depression might be avoided with this lower dose of IT morphine. Finally, after this IT morphine dose, there seems to be no need for particular supervision.

Age seems to be involved in the development of the respiratory depression after IT morphine [18]. The patients in our study had a mean age of 67.4 year. Only nine of the patients were less than 60 years of age. Likewise, the patients in previous studies had a mean age of 65-67 year in major knee surgery $[2,10,11]$. Accordingly, we recommend that lower doses of opiates be used in patients undergoing TKR. In our study, the minimum effective dose of IT morphine was $0.05 \mathrm{mg}$, and therefore respiratory depression is less likely to occur when such a very low dose of IT morphine is given, even in elderly patients.

Our study has several limitations. First, Slappendel et al [12] has examined doses as small as $0.025 \mathrm{mg}$ IT morphine. Our work did not include patients who received doses smaller than $0.05 \mathrm{mg}$ IT morphine. Therefore, we can not show any minimum dose rather than $0.05 \mathrm{mg}$ IT morphine. Second, Bailey et al [16] found that respiratory rate is not reliable as an indicator of significant respiratory depression after IT morphine and only pulse oximetry reliably detected inadequate oxygenation. We measured respiratory rates intermittently and pulse oximetry has not been used for continuous assessment of arterial oxygen saturation on the orthopedic ward; thus, significant hypoxemia between observations cannot be excluded. It is possible that episodes of subclinical respiratory depression may have occurred and gone undetected. Third, $10 \mathrm{mg}$ metoclopramide IV and $8 \mathrm{mg}$ ondansetron IV were routinely prescribed by the orthopedic surgeon for antiemetic medications to all patients for the first two days. These antiemetics should have been limited to evaluate the incidence of PONV by IT morphine used. Fourth, unfortunately, the doses of bupivacaine and volumes of injectate used for SA in all groups were not standardized, since the doses of bupivacaine used were left to the discretion of the attending anesthesiologist. The median doses (range) of spinal bupivacaine used were $9.0(6-13), 6.0$ $(5-8), 7.0(5-9), 6.5(6-8)$, and $6.0(6-8) \mathrm{mg}$ in 0.0 , $0.05,0.1,0.15,0.2 \mathrm{mg}$ IT morphine groups, respectively.

In conclusion, our study has shown that IT morphine from 0.05 to $0.2 \mathrm{mg}$ provided comparable and effective postoperative analgesia when combined with continuous 3-in-1 block after TKR. However, the incidence of vomiting was significantly more frequent with $0.1-0.2 \mathrm{mg}$ IT morphine groups compared with control, and there was a significant trend towards an increasing number of patients who required antipruritic therapy as IT morphine dose increased. Therefore, 0.05 mg IT morphine would appear to provide the optimal balance between pain relief and adverse effects following TKR.

\section{ACKNOWLEDGEMENTS}

Europian Society of Regional Anesthesia, 2007, Valencia, Spain.

\section{REFERENCES}

1. Pang WW, Hsu TC, Tung CC, Hung CP, Chang DP, Huang MH. Is total knee replacement more painful than total hip replacement? Acta Anaesthesiol Sin 2000; 38: 143-8.

2. Rathmell JP, Pino CA, Taylor R, Patrin T, Viani BA. Intrathecal morphine for postoperative analgesia: a randomized, controlled, dose-ranging study after hip and knee arthroplasty. Anesth Analg 2003; 97: 1452-7.

3. Hwang HS, Kim HH, Shin JW, Leem JG, Lee C, Yang HS, et al. Comparison of analgesic requirements for postoperative pain control in patients undergoing major orthopedic surgery. Korean J Pain 2004; 17: 228-33.

4. Edwards ND, Wright EM. Continuous low-dose 3-in-1 nerve 
blockade for postoperative pain relief after total knee replacement. Anesth Analg 1992; 75: 265-7.

5. Singelyn FJ, Deyaert M, Joris D, Pendeville E, Gouverneur JM. Effects of intravenous patient-controlled analgesia with morphine, continuous epidural analgesia, and continuous three-in-one block on postoperative pain and knee rehabilitation after unilateral total knee arthroplasty. Anesth Analg 1998; 87: 88-92.

6. Horlocker TT, Wedel DJ. Neuraxial block and low-molecular-weight heparin: balancing perioperative analgesia and thromboprophylaxis. Reg Anesth Pain Med 1998; 23(6 Suppl 2): 164-77.

7. Park CK, Cho JH, Cho CK, Kim YJ. Comparison of continuous three-in-one block and intravenous patient-controlled analgesia for postoperative pain after total knee replacement. Korean J Anesthesiol 2006; 51: 76-81.

8. Ben-David B, Schmalenberger K, Chelly JE. Analgesia after total knee arthroplasty: is continuous sciatic blockade needed in addition to continuous femoral blockade? Anesth Analg 2004; 98: 747-9.

9. Macalou D, Trueck S, Meuret P, Heck M, Vial F, Ouologuem S, et al. Postoperative analgesia after total knee replacement: the effect of an obturator nerve block added to the femoral 3-in-1 nerve block. Anesth Analg 2004; 99: 251-4.

10. Park CK, Cho CK, Shin HH, Cho JH. The effect of intrathecal morphine added to continuous femoral 3-in-1 nerve block for analgesia after total knee replacement. Korean J Anesthesiol 2008; 54: 544-51.

11. Tarkkila P, Tuominen M, Huhtala J, Lindgren L. Comparison of intrathecal morphine and continuous femoral 3-in-1 block for pain after major knee surgery under spinal anaesthesia. Eur $\mathbf{J}$ Anaesthesiol 1998; 15: 6-9.

12. Slappendel R, Weber EW, Dirksen R, Gielen MJ, van Limbeek J. Optimization of the dose of intrathecal morphine in total hip surgery: a dose-finding study. Anesth Analg 1999; 88: 822-6.

13. Murphy PM, Stack D, Kinirons B, Laffey JG. Optimizing the dose of intrathecal morphine in older patients undergoing hip arthroplasty. Anesth Analg 2003; 97: 1709-15.

14. Jacobson L, Chabal C, Brody MC. A dose-response study of intrathecal morphine: efficacy, duration, optimal dose, and side effects. Anesth Analg 1988; 67: 1082-8.

15. Glass PS. Respiratory depression following only $0.4 \mathrm{mg}$ of intrathecal morphine. Anesthesiology 1984; 60: 256-7.

16. Bailey PL, Rhondeau S, Schafer PG, Lu JK, Timmins BS, Foster $\mathrm{W}$, et al. Dose-response pharmacology of intrathecal morphine in human volunteers. Anesthesiology 1993; 79: 49-59.

17. Cole PJ, Craske DA, Wheatley RG. Efficacy and respiratory effects of low-dose spinal morphine for postoperative analgesia following knee arthroplasty. Br J Anaesth 2000; 85: 233-7.

18. Gjessing J, Tomlin PJ. Postoperative pain control with intrathecal morphine. Anaesthesia 1981; 36: 268-76.

19. Cardoso MM, Carvalho JC, Amaro AR, Prado AA, Cappelli EL. Small doses of intrathecal morphine combined with systemic diclofenac for postoperative pain control after cesarean delivery. Anesth Analg 1998; 86: 538-41.

20. Yaksh TL, Wilson PR, Kaiko RF, Inturrisi CE. Analgesia produced by a spinal action of morphine and effects upon parturition in the rat. Anesthesiology 1979; 51: 386-92.

21. Kalso E. Effects of intrathecal morphine, injected with bupivacaine, on pain after orthopaedic surgery. Br J Anaesth 1983; 55: 415-22.

22. Rathmell JP, Lair TR, Nauman B. The role of intrathecal drugs in the treatment of acute pain. Anesth Analg 2005; 101(Suppl 5): S $30-43$.

23. Uchiyama A, Ueyama H, Nakano S, Nishimura M, Tashiro C. Low dose intrathecal morphine and pain relief following caesarean section. Int J Obstet Anesth 1994; 3: 87-91.

24. Ashburn MA. Postoperative pain. In: Bonica's management of pain. 3rd ed. Edited by Loeser JD: Philadelphia, Lippincott Williams \& Wilkins. 2001, p 769.

25. Chadwick HS, Ready LB. Intrathecal and epidural morphine sulfate for post-cesarean analgesia--a clinical comparison. Anesthesiology 1988; 68: 925-9.

26. Wilder-Smith $\mathrm{OH}$, Arendt-Nielsen L. Postoperative hyperalgesia: its clinical importance and relevance. Anesthesiology 2006; 104: 601-7.

27. De Kock MF, Lavand'homme PM. The clinical role of NMDA receptor antagonists for the treatment of postoperative pain. Best Pract Res Clin Anaesthesiol 2007; 21: 85-98.

28. Kuipers PW, Kamphuis ET, van Venrooij GE, van Roy JP, Ionescu TI, Knape JT, et al. Intrathecal opioids and lower urinary tract function: a urodynamic evaluation. Anesthesiology 2004; 100: 1497-503.

29. Ready LB, Loper KA, Nessly M, Wild L. Postoperative epidural morphine is safe on surgical wards. Anesthesiology 1991; 75: 452-6.

30. Schaer H, Baasch K, Prochacka K. Intrathecal morphine for postoperative pain. Anaesthesist 1992; 41: 689-93. 